EESTI NSV TEADUSTE AKADEEMIA TOIMETISED.

FUUSIKA * MATEMAATIKA

ИЗВЕСТИЯ АКАДЕМИИ НАУК ЭСТОНСКОН ССР. ФИЗИКА МАТЕМАТИКА

PROCEEDINGS OF THE ACADEMY OF SCIENCES OF THE ESTONIAN SSR.

PHYSICS * MATHEMATICS

$1985,34,1$

УдК 535.37

M. СЕЛЬГ

\title{
КОЛЕБАТЕЛЬНАЯ РЕЛАКСАЦИЯ КВАЗИМОЛЕКУЛ В КРИСТАЛЛАХ ЭЛЕМЕНТОВ НУЛЕВОЙ ГРУППЫ
}

\author{
(Представил К. К. Ребане)
}

\section{1. Введение}

Основные центры собственной люминесценции в кристаллах элементов нулевой группы периодической системы - возбужденные квазимолекулы $\mathrm{R}_{2}{ }^{*}$ ( $\mathrm{R}$ - символ соответствующего химического элемента) отличаются от большинства известных центров свечения в кристаллах сравнительно медленной колебательной релаксацией. Поэтому кроме обычной люминесценции (ОЛ) этих центров удается зарегистрировать и свечение в ходе колебательной релаксации, т. е. горячую люминесценцию (ГЛ) даже в стационарных условиях возбуждения $\left[{ }^{1,2}\right]$. Центр $\mathrm{R}_{2}{ }^{*}$, с одной стороны, представляет собой экситон, локализованный на двух соседних узлах кристаллической решетки *. С другой стороны, он является кристаллическим аналогом возбужденной двухатомной молекулы инертного газа (эксимера). Кванты внутримолекулярных колебаний $\mathrm{R}_{2}{ }^{*}$ значительно превышают предельную энергию фононов данного кристалла (в случае центра $\mathrm{Ne}_{2}{ }^{*}$ почти в десять раз), что в конечном счете и является основным фактором, замедляющим колебательную релаксацию.

Особый интерес представляет выяснение механизма возникновения ГЛ в спектре кристалла самого легкого (не считая Не) инертного элемента $\mathrm{Ne}$.

ГЛ квазимолекул $\mathrm{Ne}_{2}{ }^{*}$ в твердом $\mathrm{Ne}$ была с достоверностью зарегистрирована уже в 1975 г. $\left[^{1}\right]$ (рис. 1). Несмотря на это, механизм ее возникновения был выяснен лишь 4-5 лет спустя, когда для интерпретации этой ГЛ была использована теория спектров резонансного вторичного свечения примесных центров кристаллов, разработанная в $[3,4]$. Для вычисления суммарных спектров ОЛ и ГЛ квазимолекул $\mathrm{R}_{2}{ }^{*}$ эта теория была применена в условиях стационарного нерезонансного возбуждения $\left[{ }^{5,6}\right]$. Таким образом расчетным путем удалось не только восстановить экспериментальный спектр свечения квазимолекул $\mathrm{Ne}_{2}{ }^{*}$ (см. рис. 1), но и установить наличие ГЛ в спектрах свечения квазимолекул $\mathrm{Xe}_{2}{ }^{*}$ и $\mathrm{Ar}_{2}{ }^{*}$ (вероятно, ГЛ содержит и спектр свечения $\mathrm{Kr}_{2}{ }^{*}$, однако этот случай пока никем не исследован). В теории имеются два подгоночных параметра: $\gamma$ - константа радиационного затухания (отметим, что в случае $\mathrm{Ne}_{2}{ }^{*}$ экспериментальные работы по определению $\gamma$ практически отсутствуют) и $\Gamma-$ константа затухания внутримолекулярного (локального) колебания (для центров $\mathrm{R}_{2}{ }^{*}$

\footnotetext{
* Напомним, что все инертные элементы (кроме Не, который в данной работе не рассматривается) при кристаллизации образуют ГЦК-решетку с одним атомом в элементарной ячейке. Поэтому спектр их кристаллических колебаний содержит только акустические ветви.
} 


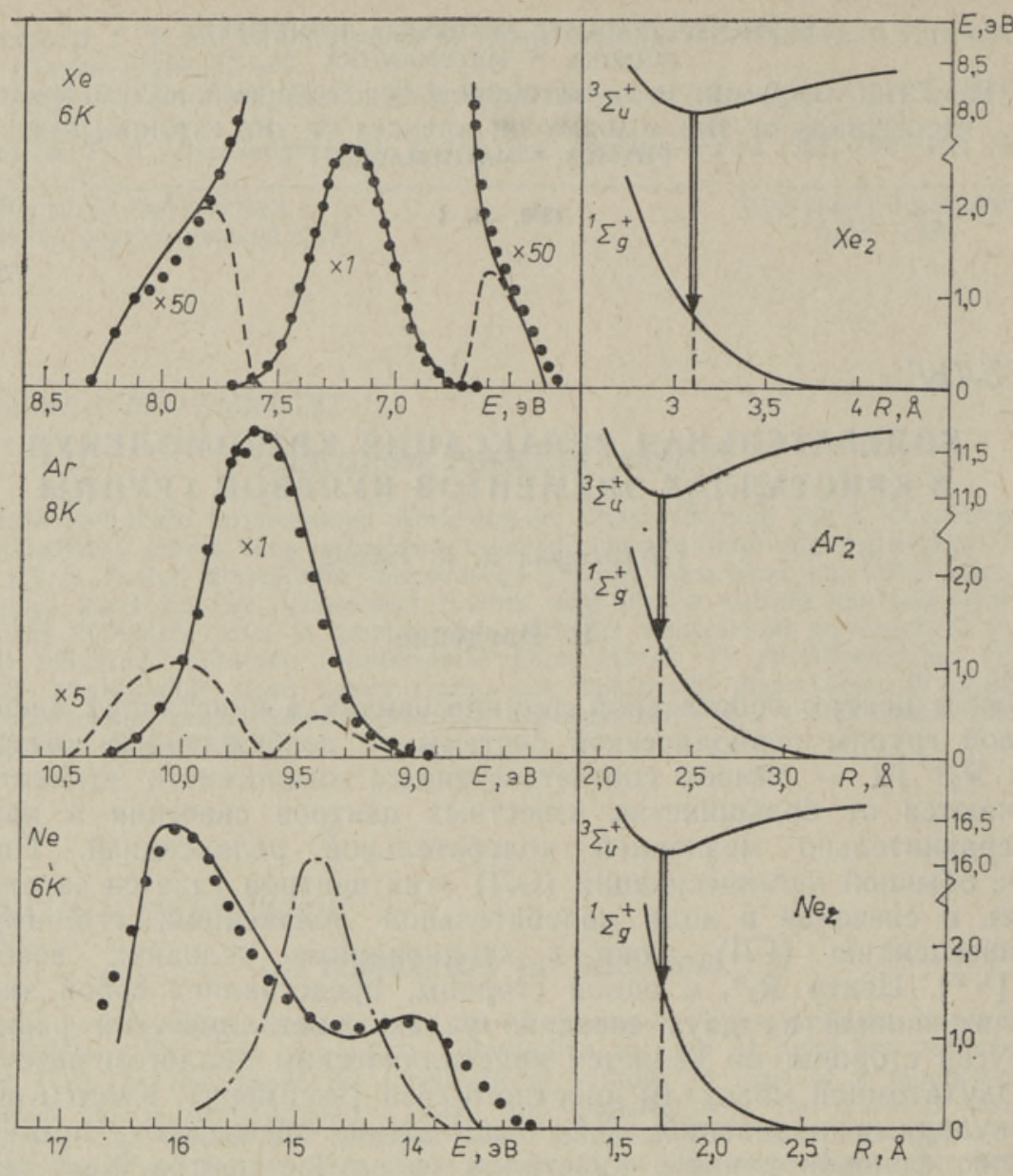

Рис. 1. экспериментальные (ㅇ) и расчетные (-) спектры свечения центров $\mathrm{R}_{2}{ }^{*}$ в кристаллах инертных элементов $\left[{ }^{6}\right]$ вместе с соответствующими кривыми потенциальной энергии. Экспериментальный спектр $\mathrm{Ne}_{2}{ }^{*}$ заимствован из [1], ГЛ $\mathrm{Ar}_{2}{ }^{*}$ и $\mathrm{Xe}_{2}{ }^{*}$ выделена пунктиром. Для сравнения приведен соответствующий спектр люминесценции газового $\mathrm{Ne}(-\cdot-)$ [ $\left.{ }^{19}\right]$.

можно применять модель затухающего осциллятора Морзе [7]), которая вводится феноменологически. Форма расчетных спектров существенно зависит от соотношения между $\gamma$ и $\Gamma$. Расчетный спектр для $\mathrm{Ne}_{2}{ }^{*}$ на рис. 1 получен при $\gamma=1,5 \Gamma$, т. е. когда время колебательной релаксации превышает радиационное время жизни центра. Поэтому свечение квазимолекул $\mathrm{Ne}_{2}{ }^{*}$ почти целиком состоит из ГЛ (доля ГЛ в єлучае центра $\mathrm{Xe}_{2}{ }^{*}$ порядка $1 \%$, а в случае $\mathrm{Ar}_{2}{ }^{*}-10 \%$ от общей светосуммы [6]).

Описанный вариант теории, который применен в $\left[{ }^{5,6}\right]$, позволяет рассчитать только огибающую спектра люминесценции. В принципе, однако, спектр может иметь и колебательную структуру, о наблюдении которой в случае центра $\mathrm{Ne}_{2}{ }^{*}$ недавно сообщалось [ $\left.{ }^{8}\right]$. Остается пока открытым и вопрос об абсолютных значениях $\gamma$ и Г. Дело в том, что форма расчетных спектров при постоянном отношении $\gamma / \Gamma$ слабо зависит от их абсолютных значений, если оба параметра малы по сравнению с частотой локального колебания.

В данной работе ставилась цель разработать микроскопическую 
теорию колебательной релаксации квазимолекул типа $\mathrm{R}_{2}{ }^{*}$ в кристаллах, т. е. прежде всего рассчитать вероятности переходов между.соседними колебательными уровнями центров $\mathrm{R}_{2}$ *

\section{2. Модель}

Будем исходить из следующего гамильтониана для взаимодействующей системы локального осциллятора-квазимолекулы и набора кристаллических осцилляторов

$$
\begin{aligned}
H & =\frac{\Omega}{2}\left(z^{2}-\frac{\partial^{2}}{\partial z^{2}}\right)+\sum_{\mathrm{k} r} \frac{\omega \mathrm{k} r}{2}\left(q_{\mathrm{k} r} q_{-\mathrm{k} r}-\frac{\partial^{2}}{\partial q_{\mathrm{k} r} \partial_{-\mathrm{k} r}}\right)+ \\
& +\sum_{\mathrm{k} r, \mathrm{k}^{\prime} r^{\prime}} F_{\mathrm{k} r, \mathrm{k}^{\prime} r^{\prime}} z q_{\mathrm{k} r} q_{\mathrm{k}^{\prime} r^{\prime}}+\sum_{\mathrm{k} r} d_{\mathrm{k} r} z^{2} q_{\mathrm{k} r}+ \\
+ & \sum_{\mathrm{k} r, \mathrm{k}^{\prime} r^{\prime}, \mathrm{k}^{\prime \prime} r^{\prime \prime}} B_{\mathrm{k} r, \mathrm{k}^{\prime} r^{\prime}, \mathrm{k}^{\prime \prime} r^{\prime \prime}} q_{\mathrm{k} r} q_{\mathrm{k}^{\prime} r^{\prime}} q_{\mathrm{k}^{\prime \prime} r^{\prime \prime}} .
\end{aligned}
$$

Здесь $\Omega$ и $z$ - частота и координата локального осциллятора, $\omega_{\mathrm{k} r}$ и $q_{\mathrm{k} r}$ - то же для кристаллических осцилляторов (k - квазиимпульс, $r=1,2,3$ - номер ветви колебаний). Используется система единиц, где $\hbar=1$. Последний член в гамильтониане учитывает ангармонизм кристаллических колебаний, что особенно для кристалла $\mathrm{Ne}$ довольно существенно. Следует отметить, что к (1) можно добавить члены, описывающие фонон-фононное взаимодействие более высокого порядка, т. е. процессы с участием более чем трех фононов. Основные выводы данной работы при этом остаются в силе.

В случае всех кристаллов инертных элементов можно считать выполненным условие $\Omega \gg \omega_{\mathrm{k} r}$. Это позволяет ввести т. н. двойное адиабатическое приближение $\left[{ }^{9}\right]$, в котором каждому колебательному уровню локального осциллятора соответствует адиабатическая поверхность в пространстве более медленных кристаллических колебаний**. Переходы между этими поверхностями осуществляются с помощью оператора неадиабатичности $L$, который в данном случае дается следующей формулой:

$$
\begin{aligned}
L_{\varphi_{n}}(z, q) X_{n}(q) & =-\frac{1}{2} \sum_{x} \omega_{x}\left\{X_{n}(q) \frac{\partial^{2} \varphi_{n}(z, q)}{\partial q_{x} \partial q_{-x}}+\right. \\
& \left.+2 \frac{\partial X_{n}(q)}{\partial q_{-x}} \cdot \frac{\partial \varphi_{n}(z, q)}{\partial q_{x}}\right\}
\end{aligned}
$$

Здесь и в дальнейшем используется сокращенное обозначение индексов - $x \equiv \mathrm{k} r ; n-$ номер исходного колебательного состояния локального осциллятора; $q$ обозначает совокупность кристаллических координат; $\varphi_{n}$ и $X_{n}$ - адиабатически разделенные волновые функции «быстрой» и «медленной» подсистем.

Нас интересует переход $n \rightarrow n-1$ в локальном осцилляторе. Поэтому определим новый оператор $M_{n}$, который будет действовать только в пространстве кристаллических координат

$$
M_{n} X_{n}(q) \equiv \int d z \varphi_{n-1}^{*}(z, q) L\left[\varphi_{n}(z, q) X_{n}(q)\right] .
$$

Перейдя к обозначениям Дирака для матричных элементов, имеем

\footnotetext{
** Применение двойного адиабатического приближения, конечно, не означает отказа от обычного адиабатического приближения, т.е. по опношению к электронам движения локального осциллятора остается адиабатически медленным.
} 


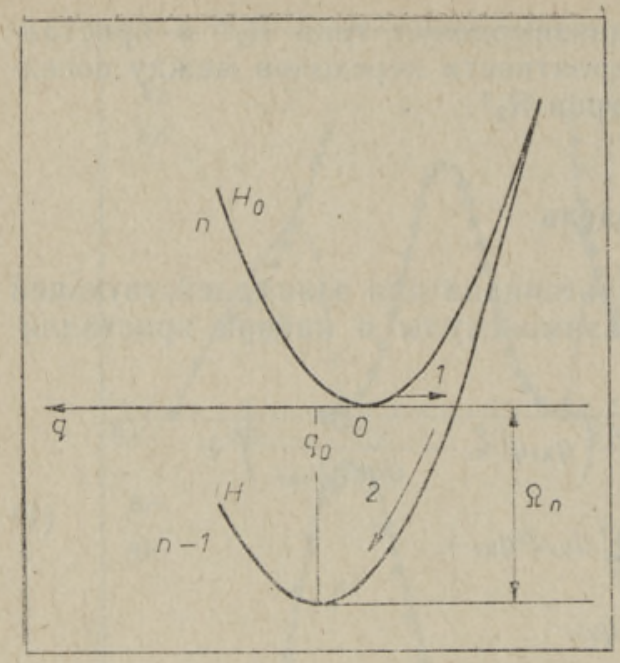

Рис. 2. Двухэтапная модель колебатель. ной релаксащии: 1 - туннелирование между адиабатическими поверхностями $\left(n, H_{0}\right) \rightarrow(n-1, H) ; 2$ - релаксация фононной подсистемы.

$$
\begin{aligned}
M_{n}= & -\frac{1}{2} \sum_{x} \omega_{x}\left\{\left\langle n-1\left|\frac{\partial^{2}}{\partial q_{x} \partial q_{-x}}\right| n\right\rangle+\right. \\
& \left.+2\left\langle n-1\left|\frac{\partial}{\partial q_{-x}}\right| n\right\rangle \frac{\partial}{\partial q_{x}}\right\} .
\end{aligned}
$$

Учитывая далее вид гамильтониана (1) и считая в нем $\sum_{j} d_{j} q_{j} \ll \frac{\Omega}{2}$, получим окончательно

$$
M_{n}=-\sqrt{\frac{n}{2}} \cdot \frac{1}{\Omega_{n}}\left\{-\sum_{x} \omega_{x} F_{x-x}+2 \sum_{x, x^{\prime}} \omega_{x} F_{x x^{\prime}} q_{x^{\prime}} \frac{\partial}{\partial q_{-x}}\right\},
$$

где $\Omega_{n}$ - расстояние между колебательными уровнями с номерами $n$ и $n-1$.

В рассматриваемой модели один элементарный акт на пути колебательной релаксации состоит как бы из двух частей [ $\left.{ }^{10}\right]$ :

1) туннелирования между адиабатическими поверхностями,

2) релаксации фононной подсистемы, т. е. перехода фононов в равновесие с новым колебательным состоянием локального осциллятора (рис. 2).

Необходимо отметить, что данный подход к проблеме колебательной релаксации локального осциллятора принципиально отличается от такового в $[11,12]$, где использовалась модель «жесткой среды», т. е. предполагалось, что фононы остаются в тепловом равновесии в течение всего процесса релаксации (в таком случае должен равняться нулю и сдвиг равновесия на рис. 2). Кроме того, использовалось т. н. приближение беспорядочных фаз, в том смысле, что произведение бозевских операторов рождения и уничтожения фононов заменялось их тепловым средним (напр., $a_{x}{ }^{+} a_{x} \rightarrow \delta_{x x} \bar{n}_{x}$, и т. д.). Не будем здесь обсуждать корректность этих приближений применительно к центрам $\mathrm{R}_{2}{ }^{*}$ в кристаллах инертных элементов. Ясно, однако, что при таком подходе учитываются только т.н. вертикальные процессы превращения кванта локального колебания в подходящее число фононов (или наоборот). В случае центра $\mathrm{Ne}_{2}{ }^{*}$ число фононов, участвующих в таком процессе, будет порядка десяти согласно закону сохранения энергии. Поэтому, применяя такой подход, мы должны были бы, по существу, оставаться на уровне качественных рассуждений, так как теории для 
описания процессов такого высокого порядка пока не разработано, о чем сказано и в $\left[{ }^{12}\right]$.

Вернемся теперь к нашей модели. Вероятность туннелирования между соседними адиабатическими поверхностями можно рассчитать. по известной формуле нестационарной теории возмущений первого порядка

$$
W=2 \pi \sum_{i} n_{i} \sum_{f}\left|\left\langle f\left|M_{n}\right| i\right\rangle\right|^{2} \delta\left(E_{i}-E_{f}\right) .
$$

Здесь $i$ и $f$ - соответственно начальное и конечное состояния совокупности кристаллических осцилляторов, $n_{i}=e^{-E_{l} / k_{B} T} / \sum_{i} e^{-E_{l} / k_{B} T}, \quad E_{i}$ и $E_{f}$ - полные энергии системы в начальном (связанном с адиабатической поверхностью номера $n$ ) и конечном $(n-1)$ состояниях соответственно.

Учитывая структуру гамильтониана (1), формулу (5) можно преобразовать

$$
W_{n \rightarrow n-1}=\int_{-\infty}^{\infty} e^{-i \Omega_{n} t}\left\langle T M_{n}^{+} e^{L} M_{n}(t)\right\rangle d t,
$$

где $T$ - оператор хронологического упорядочения (располагает операторы с временным аргументом $t$ правее от независящих от времени операторов); $L=\nabla-\nabla(t), \quad$ где $\nabla=\sum_{x} q_{0}^{x} \frac{\partial}{\partial q_{x}}-$ оператор сдвига; $q_{0}^{x}=\frac{d_{-x}}{\omega_{x}} ;$ гамильтониан $H$ в конечном $(n-1)$ состоянии локального осциллятора выражается через исходный (в состоянии $n$ ) гамильтониан $H_{0}$ как $\quad H=-\Omega_{n}+e^{\nabla} H_{0} e^{-\nabla} ; \quad A(t)=e^{i t H_{0}} A e^{-i t H_{0}} ;$ $\langle A\rangle=S p\left(e^{-H_{0} / k_{B} T} A\right) / S p\left(e^{-H_{V} / k_{B} T}\right)$.

\section{3. Вероятности переходов между колебательными уровнями}

Для вычисления коррелятора в $\left(5^{\prime}\right)$ нам прежде всего необходимо уточнить ряд множителей $F_{\mathrm{k} r, \mathrm{k}^{\prime} r^{\prime}}$ и $d_{\mathrm{k} r}$ в гамильтониане (1). Разложим потенциальную энергию кристалла в ряд по реальным смещениям $\left(u_{n \alpha}\right)$ атомов, затем перейдем к комплексным нормальным координатам согласно формуле $\left[{ }^{13}\right]$

$$
u_{\mathrm{n} \alpha}=\sum_{\mathrm{k} r} \frac{\dot{e}_{\alpha}^{r}(\mathrm{k}) e^{i \mathrm{kn}}}{\sqrt{m \omega_{\mathrm{k} r}}} q_{\mathrm{k} r}
$$

$\left(e_{\alpha}^{r}-\right.$ проекция векторов поляризации фононов, $m$ и $N$ - масса и число атомов) и сравним результат с выражением для потенциальной энергии в (1). Тогда с учетом только парных взаимодействий между атомами ***, описываемых потенциалом $W(r)$, получим

$$
d_{\mathrm{k} r}=\frac{1}{12 m \Omega} \sum_{\mathrm{n} \alpha} W^{\prime \prime \prime}\left(r_{\mathrm{n}}^{0}\right) \cos \alpha_{\mathrm{n}} \cos ^{2} z_{\mathrm{n}} \frac{e_{\alpha}^{r}(\mathrm{k}) e^{i \mathrm{kn}}}{\sqrt{m N_{\mathrm{k} r}}}
$$

где $r_{\mathrm{n}}{ }^{0}-$ равновесное расстояние между п-м атомом и одним из двух компонент квазимолекулы $\mathrm{R}_{2} *$. Ось $z$ прямоугольной системы координат совпадает с осью квазимолекулы; $\alpha_{n}$ - угол между вектором $r_{\mathrm{n}}{ }^{0}$ и осью $\alpha$.

*** Вклад трехчастичных взанмодействий в когезионную энергию кристалла $\mathrm{Ne}$ составляет $3-4 \%$ [14]. 
Аналогично

$$
\begin{aligned}
& F_{\mathrm{k} r, \mathrm{k}^{\prime} r^{\prime}}=-\frac{1}{\sqrt{72 m \Omega}} \sum_{\mathrm{n}, \alpha, \beta} W^{\prime \prime \prime}\left(r_{\mathrm{n}}^{0}\right) \cos \alpha_{\mathrm{n}} \cos \beta_{\mathrm{n}} \cos z_{\mathrm{n}} \times \\
& \times \frac{e_{\alpha}^{r}(\mathrm{k}) e_{\beta}^{r^{\prime}}\left(\mathrm{k}^{\prime}\right)}{m N \sqrt{\omega_{\mathrm{k} r} \omega \mathrm{k}^{\prime} r^{\prime}}} e^{i\left(\mathrm{k}+\mathrm{k}^{\prime}\right) \mathrm{n}} .
\end{aligned}
$$

В (8) опущен член, содержащий вторые производные $\mathrm{W}^{\prime \prime}$, который по сравнению с выписанным членом имеет порядок малости $\left(\omega_{D} / \Omega\right)^{2}$.

Попытаемся теперь вычислить важный параметр $B \equiv \frac{1}{2} \sum_{\mathrm{k} r} q_{0}^{\mathrm{k} r} q_{0}^{-\mathrm{k} r}$ (безразмерные стоксовые потери). Для этого заметим, что формулу (7) можно переписать следующим образом

$$
d_{\mathrm{k} r}=\frac{1}{12 m \Omega} \sum_{\mathrm{n} \alpha}^{\prime} W^{\prime \prime \prime}\left(r_{\mathrm{n}}^{0}\right) \cos \alpha_{\mathrm{n}} \cos ^{2} z_{\mathrm{n}} e^{i \mathrm{kn}}\left(1-e^{-2 i k_{\alpha} n_{\alpha}}\right) \frac{e_{\alpha}^{r}(\mathrm{k})}{\sqrt{m N_{\omega \mathrm{k} r}}},
$$

где штрих у знака суммы означает, что при фиксированном $\alpha$ суммирование производится в полупространстве, определенном (например) условием $\cos \alpha_{\mathrm{n}}>0$.

Для простоты при вычислении параметра $B$ будем учитывать лишь ближайшие окружающие $\mathrm{R}_{2}{ }^{*}$ атомы (в полупространстве $\cos \alpha_{\mathrm{n}}>0$ таких атомов четыре). Тогда, используя прнближение $\omega_{\mathrm{k} r} \approx \omega_{\mathrm{k}} \approx u k$ (до некоторого предельного значения $k_{m}$ ), с учетом $\left(7^{\prime}\right)$ имеем

$$
B=\frac{1}{72 m^{3} \Omega^{2} N} \sum_{\mathrm{k}, \mathrm{n}, \mathrm{n}_{1}, \alpha}^{\prime \prime} \frac{\left[W^{\prime \prime \prime}\left(r_{\mathrm{n}}^{0}\right)\right]^{2}}{\omega_{\mathrm{k}}^{3}} \cos ^{2} \alpha_{\mathrm{n}} \cos ^{4} z_{\mathrm{n}} e^{i \mathrm{k}\left(\mathrm{n}-\mathrm{n}_{\mathrm{n}}\right)} \sin ^{2}\left(k_{\alpha} n_{\alpha}\right) .
$$

Двойной штрих у знака суммы означает здесь, что векторы $\mathrm{n}$ и $\mathrm{n}_{1}$ соответствуют атомам из ближайшего окружения $\mathrm{R}_{2}{ }^{*}$. Кроме того, мы учли, что $W^{\prime \prime \prime}\left(r_{\mathrm{n}}^{0}\right)=W^{\prime \prime \prime}\left(r_{\mathrm{n}_{1}}^{0}\right)$ и $\cos \alpha_{\mathrm{n}}=\cos \alpha_{\mathrm{n}_{1}}$.

Переходя в последней формуле от суммирования в k-пространстве к интегрированию согласно формуле $\sum_{\mathrm{k}} \ldots=\frac{N v}{(2 \pi)^{3}} \int \ldots d^{3} \mathrm{k}$, где $v$ - объем элементарной ячейки, и, введя цилиндрические координаты $k_{\alpha}, \varrho \equiv \sqrt{k^{2}-k_{\alpha}^{2}}, \varphi$ после интегрирования по $\varphi$ на интервале $(0,2 \pi)$, можно получить

$$
\begin{gathered}
B=\frac{\pi}{144 m^{3} \Omega^{2} \omega_{D}^{3}} \sum_{\mathrm{n}, \mathrm{n}_{1}, \alpha}^{\prime \prime}\left[W^{\prime \prime \prime}\left(r_{\mathrm{n}}^{0}\right)\right]^{2} \cos ^{2} \alpha_{\mathrm{n}} \cos ^{4} z_{\mathrm{n}} \times \\
\times \int_{0}^{k_{m}} d k \int_{-k}^{k} d k_{\alpha} \frac{\sin ^{2}\left(k_{\alpha} n_{\alpha}\right)}{k^{2}} \sum_{l=0}^{\infty}\left(-\frac{k^{2}-k_{\alpha}^{2}}{4}\right)^{l} \cdot \frac{\left(t^{2}-t_{\alpha}^{2}\right)^{l}}{(l !)^{2}} .
\end{gathered}
$$

Здесь введен вектор $t\left(t_{x}, t_{y}, t_{z}\right) \equiv \mathrm{n}-\mathrm{n}_{1} ; \omega_{D}-$ частота Дебая.

По формуле (9) можно рассчитать $B$ с любой требуемой точностью (в рамках предложенной выше модели изотропного кристалла). С точностью до слагаемых с $l=2$ двойной интеграл в (9) равен

$$
S_{1}\left(2 k_{m} n_{\alpha}\right)-\left(\frac{t^{2} k_{m}^{2}}{12}-\frac{t^{4} k_{m}^{4}}{480}\right)+
$$




$$
\begin{gathered}
+\left(1-\frac{t^{2}}{8 n_{\alpha}^{2}}+\frac{3 t^{4}}{128 n_{\alpha}^{4}}\right)\left[\frac{\sin \left(2 k_{m} n_{\alpha}\right)}{2 k_{m} n_{\alpha}}-1\right]+F\left(k_{m} n_{\alpha}, t\right) \approx \\
\approx S_{1}\left(2 k_{m} n_{\alpha}\right), \\
{ }^{2 k_{m} n_{\alpha}}
\end{gathered}
$$

где $F\left(k_{m} n_{\alpha}, t\right) \ll S_{1}\left(2 k_{m} n_{\alpha}\right)=\int_{0}^{\alpha} \frac{1-\cos u}{u} d u$.

Поэтому приближенно можно написать

$$
B \approx \frac{\pi}{36 m^{3} \Omega^{2} \omega_{D}^{3}} \sum_{\mathrm{n} \alpha}^{\prime}\left[W^{\prime \prime \prime}\left(r_{\mathrm{n}}^{0}\right)\right]^{2} \cos ^{2} \alpha_{\mathrm{n}} \cos ^{4} z_{\mathrm{n}} S_{1}\left(2 k_{m} n_{\alpha}\right) .
$$

Вид парного потенциала $W(r)$, описывающего взаимодействие между одной из компонент квазимолекулы $\mathrm{R}_{2}$ * и атомом в кристалле, в случае атомарных криокристаллов пока не установлен. На расстояниях, существенно превышающих равновесное расстояние между компонентами $\mathrm{R}_{2}{ }^{*}$, с некоторым основанием $\left[{ }^{6,15}\right] W(r)$ можно заменить потенциалом невозбужденной молекулы $\mathrm{R}_{2}$, в случае $\mathrm{Ne}_{2}$ хорошо аппроксимируемого потенциалом Морзе

$$
W(r)=D\left[e^{-\beta\left(r-r_{0}\right)}-1\right]^{2}
$$

с параметрами $D=3,7$ мэВ, $\beta=2,02 \AA^{-1}, r_{0}=3,10 \AA\left[{ }^{14}\right]$.

Для вычисления параметра $B$ мы еще должны знать взаимное расположение $\mathrm{R}_{2}{ }^{*}$ и окружающих атомов. В [ $\left.{ }^{6}\right]$ предложены две возможные конфигурации: 1) конфигурация локализованного на вакансии экситона, 2) конфигурация автолокализованного экситона. В обоих случаях с помощью $\left(9^{\prime}\right)$ и (10) можно получить практически одинаковый результат $B \approx 4 \cdot 10^{-5}$, т. е. $B \ll 1$. Поэтому можно утверждать, что в случае $\mathrm{Ne}$ оператор сдвига $\nabla=\sum_{x} q_{0}^{x} \frac{\partial}{\partial q_{x}}$ практически равен нулю, а следовательно, и оператор $L=\nabla-\nabla(t) \approx 0$. Учитывая это, нетрудно произвести интегрирование в $\left(5^{\prime}\right)$. С учетом (4) и (8) имеем

$$
W_{n \rightarrow n-1}=\frac{n V^{2}}{72 m^{3} \Omega_{n}^{3}(\Omega-2 \bar{\omega})^{2}(2 \pi)^{6}} \sum_{n, n^{\prime}, \alpha, \beta} W^{\prime \prime \prime}\left(r_{n}^{0}\right) W^{\prime \prime \prime}\left(r_{n^{\prime}}^{0}\right) \times
$$

$\times \cos \alpha_{\mathrm{n}} \cos \alpha_{\mathrm{n}^{\prime}} \cos \beta_{\mathrm{n}} \cos \beta_{\mathrm{n}^{\prime}} \cos z_{\mathrm{n}} \cos z_{\mathrm{n}^{\prime}} \iint d^{3} \mathrm{k} d^{3} \mathrm{k}^{\prime} e^{i\left(\mathrm{k}+\mathrm{k}^{\prime}\right)\left(\mathrm{n}+\mathrm{n}^{\prime}\right)} \times$

$$
\times\left(1+\omega_{\mathrm{k}} / \omega_{\mathrm{k}^{\prime}}\right)\left(A_{\mathrm{k}}+A_{\mathrm{k}^{\prime}}\right) .
$$

Здесь $\bar{\omega}-$ некоторая средняя частота кристаллических колебаний, а появление величин $A_{\mathrm{k}} \neq 0$ целиком обусловлено ангармонизмом кристаллических колебаний. Если учитывать только кубический ангармонизм колебаний, то для этих величин можно написать следующую приближенную формулу

$A_{\mathrm{k}}=\sum_{i=1}^{\infty} \sum_{\mathrm{k}_{1}, \mathrm{k}_{2}, \ldots \mathrm{k}_{\mathrm{st}}} \alpha_{\mathrm{k}_{1} \mathrm{k}_{2} \ldots \mathrm{k}_{\mathrm{st}}}^{(i)}\left[W^{\prime \prime \prime}\left(r_{0}\right)\right]^{2 i} \delta\left(\Omega-2 \omega_{\mathrm{k}}-\omega_{\mathrm{k}_{1}}-\ldots-\omega_{\mathrm{k}_{s t}}\right)$.

Вычисление коэффициентов $\alpha_{\mathrm{k}_{1} \mathrm{k}_{2} \ldots \mathrm{k}_{s t}}^{(i)}$ в (12) представляет собой самостоятельную весьма сложную задачу, решение которой выходит за рамки данной работы. Отметим, что в случае Ne первое (и основное) отличное от нуля слагаемое в (12) соответствует значению $i=2$.

В (11) с достаточным основанием можно ограничиться учетом слагаемых с $\mathrm{n}^{\prime}=-\mathrm{n}$. Это почти очевидно в случае изо- 
тропного кристалла, когда в (11) легко произвести интегрирование по направлениям $\mathrm{k}$ и $\mathrm{k}^{\prime}$, после чего в этой формуле появились бы множители $\frac{\sin \left(k\left|\mathrm{n}+\mathrm{n}^{\prime}\right|\right) \sin \left(k^{\prime}\left|\mathrm{n}+\mathrm{n}^{\prime}\right|\right)}{k k^{\prime}\left|\mathrm{n}+\mathrm{n}^{\prime}\right|^{2}}$, быстро убывающие с ростом $\left|\mathrm{n}+\mathrm{n}^{\prime}\right|$. У реальных (анизотропных) кристаллов получаемые описанным образом множители имеют более сложный вид. Однако можно ожидать, что и они становятся быстро убывающими с ростом $\left|n+n^{\prime}\right|$.

Таким образом, используя указанное приближение, имеем окончательно

$$
W_{n \rightarrow n-1}=\frac{n A}{72 m^{3} \Omega_{n}^{3}(\Omega-2 \bar{\omega})^{2}},
$$

где

$$
\begin{aligned}
A= & \frac{v^{2}}{(2 \pi)^{6}} \sum_{\mathrm{n}}\left[W^{\prime \prime \prime}\left(r_{\mathrm{n}}^{0}\right)\right]^{2} \cos ^{2} z_{\mathrm{n}} \iint d^{3} \mathrm{k} d^{3} \mathrm{k}^{\prime}\left(1+\frac{\omega_{\mathrm{k}}}{\omega_{\mathrm{k}^{\prime}}}\right)\left(A_{\mathrm{k}}+A_{\mathrm{k}^{\prime}}\right) \approx \\
& \approx \sum_{\mathrm{n}}\left[W^{\prime \prime \prime}\left(r_{\mathrm{n}}^{0}\right)\right]^{2} \cos ^{2} z_{\mathrm{n}} \int_{0}^{\omega_{m}} d_{\omega} \int_{0}^{\omega_{m}} d \omega^{\prime} \mathrm{\varrho}(\omega) \mathrm{Q}\left(\omega^{\prime}\right)\left(1+\frac{\omega}{\omega^{\prime}}\right)\left(A \omega+A \omega^{\prime}\right),
\end{aligned}
$$

$\mathrm{\varrho}(\omega)$ - плотность числа колебаний и $\omega_{m}-$ максимальная частота в спектре кристаллических колебаний. В данной работе $A$ рассматривается как параметр теории, значение которого следует определить экспериментально. Как показано в $\left[{ }^{16}\right]$ согласие с экспериментальным спектром свечения $\mathrm{Ne}_{2}{ }^{*}$ [ $\left.{ }^{1}\right]$ наиболее хорошее при значении $A=5 \cdot 10^{-9}$ ат. ед. Используя это значение $A$, а также известное соотношение для осциллятора Морзе $\Omega_{n}=\Omega\left(1-\frac{n \Omega}{2 D_{l}}\right)$, где для $\mathrm{Ne}_{2}$ * $\Omega=51,5$ мэВ и $D_{l}=0,64$ эВ $\left[{ }^{17}\right]$ и, выбирая значение $\bar{\omega}=3,5$ мэВ (такое значение получается при усреднении величины $\left.\left(\Omega_{n}-2 \omega_{k}\right)^{-2}\right)$, можно по формуле (13) рассчитать вероятности переходов, приведенные в таблице ****:

\begin{tabular}{cccccc} 
& 1 & 2 & 3 & 4 & 5 \\
\hline$W_{n \rightarrow n-1}$ & $6,30 \cdot 10^{5}$ & $1,58 \cdot 10^{6}$ & $3,02 \cdot 10^{6}$ & $5,18 \cdot 10^{6}$ & $8,44 \cdot 10^{6}$ \\
& 6 & 7 & 8 & 9 & 10 \\
\cline { 2 - 6 } & $1,34 \cdot 10^{7}$ & $2,11 \cdot 10^{7}$ & $3,30 \cdot 10^{7}$ & $5,21 \cdot 10^{7}$ & $8,31 \cdot 10^{7}$
\end{tabular}

Мы использовали значение $D_{l}=0,64$ эВ. Это соответствует аппроксимации расчетной кривой потенциальной энергии ${ }^{3} \Sigma_{u}{ }^{+}$-состояния $\mathrm{Ne}_{2}{ }^{*}$ функцией Морзе. Такую аппроксимацию можно считать хорошей в широком интервале межъядерных расстояний. В области больших расстояний все же отклонения от кривой Морзе становятся значительными и поэтому истинный предел диссоциации, определенный в $\left[{ }^{17}\right]$, $D_{l} \approx 0,45$ эВ, чему соответствует максимальное допустимое значение $n=10$. Это учтено и при составлении таблицы.

\section{4. Заключительные замечания}

Основным результатом работы является установление такой функциональной зависимости вероятностей колебательных переходов

**** Температурная зависимость $W_{n \rightarrow n-1}(T)$ осуществляется через параметры $A_{\mathrm{k}}$. Поскольку в случае кристалла $\mathrm{Ne}$ при всякой реально достигаемой температуре эксперимента средние числа заполнения фононов $\bar{n}_{\mathrm{k}} \ll 1$, можно считать, что в этом случае величины $W_{n \rightarrow n-1}$ практически независимы от температуры. 
$W_{n \rightarrow n-1}(n)$ в медленно релаксирующих локальных центрах свечения, которая согласуется с их наблюдаемыми спектрами люминесценции (имеется в виду прежде всего центр $\mathrm{Ne}_{2}{ }^{*}$ ). При попытках построения «вертикальной» теории колебательной релаксации (см., напр., серию статей $\left.\left[{ }^{18}\right]\right)$ или же кгоризонтальной» (т. е. туннельной) теории с параметром $B \gg 1$ устанавливается слишком сильная зависимость $W_{n \rightarrow n-1}$ от номера колебательного уровня $(n)$ центра. Неизбежным следствием этого является возможность излучательного перехода практически только с одного колебательного уровня (радиационное время жизни которого сравнимо с $W_{n \rightarrow n-1}^{-1}$ ), что по крайней мере в случае $\mathrm{Ne}_{2}{ }^{*}$ противоречит эксперименту.

Автор искренне благодарен В. Хижнякову за очень полезное обсуждение результатов работы.

\section{ЛИТЕРА Т РА}

1. Schuberth, E., Creuzburg, M. Phys. status solidi (b), 71, № 2, 797-802 (1975).

2. Кинк Р. А., Лыхмус А. Э., Сельг М. В. Письма в ЖЭТФ, 28, вып. 8, 505-508 (1978).

3. Техвер И. Ю., Хижняяков В. В. Ж. эксперим. и теор. физ., 69, вып. 2, 599-610 (1975).

4. Хижняков В., Ребане И. Изв. АН ЭССР. Физ.-Матем., 26, № 3, 260-280 (1977).

5. Hizhnyakov, V., Kink, R., Selg, M., Sherman, A. Abstr. 2nd Intern. Symp. \&Ultrafast Phenomena in Spectroscopy». Reinhardsbrunn, 1980, 2, 468-472.

6. Kink, R. Lõhmus, A., Selg, M. Phys. status solidi (b), 107, № 2, 479-490 (1981).

7. Сельг М. В. Релаксация и горячая люминесценция автолокализующихся экснтонов в кристаллах инертных газов. Автореф. канд. дис. Тарту, 1981.

8. Григоращщенко О. Н., Рыбалко Ю. Н., Савченко Е. В., Фуголь И. Я. Физ. низк. температур, 8, № 8, 886-890 (1982).

9. Сияьд О. Изв. АН ЭССР. Физ.-Матем., 17, № 2, 203-211 (1968).

10. Сильд О. Н., Хижняков В. В. В кн.: Мат. междунар. семинара кИзбранные проблемы теории примесного центра кристалла». Таллин, 1972, 674.

11. Nitzan, A., Jortner, J. Mol. Phys., 25, № 3, 713-734 (1973).

12. Nitzan, A., Mukamel, S., Jortner, J. J. Chem. Phys., 60, № 10, 3929-3934 (1974).

13. Давыдов А. С. Теория твердого тела. М., «Наука», 1976.

14. Barker, J. A. In: Rare Gas Solids, 1. New York-London-Paris, Academic Press, $1976,212-264$.

15. Fugol, I. Ya., Belov, A. G., Tarasova, E. I. Solid State Commun., 32, № 9, 787790 (1979).

16. Сельг М. Изв. АН ЭССР. Физ.-Матем. (в печати).

17. Berman, M., Kaldor, U. Chem. Phys., 43, № 2, 375-383 (1979).

18. Yakhot, V., Berkowitz, M., Gerber, R. B. Chem. Phys., 10, № 1, 61-66 (1975);

Yakhot, V. Phys. status solidi (b), 76, № 2, 451-456 (1976);

Yakhot, V. Chem. Phys., 14, № 2, 441-453 (1976).

19. Leichner, P. K. Phys. Rev., A8, № 2, 815-822 (1973).
Ннститут физики
Академии наук Эстонской ССР
Поступила в редакцию
$11 / \mathrm{X} 1983$

M. SELG

\section{KVAASIMOLEKULIDE VÕNKERELAKSATSIOON NULLROHMA ELEMENTIDE KRISTALLIDES}

On esitatud teoreetiline mudel, mis sobib lokaliseeritud eksitonide (kvaasimolekulide) vōnkerelaksatsiooni kirjeldamiseks atomaarsetes krüokristallides ( $\mathrm{Xe}, \mathrm{Kr}, \mathrm{Ar}$, Ne), ja arvutatud üleminekutōenäosused kiirgustsentri $\mathrm{Ne}_{2}{ }^{*}$ naabervõnkenivoode vahel. Osutub, et tsentri $\mathrm{Ne}_{2}{ }^{*}$ arvutatud vōnkerelaksatsiooniaeg on vōrreldav tema kiirgusliku èlueaga. See on kooskõlas eksperimentaalse faktiga, et lõviosa kvaasimolekuli $\mathrm{Ne}_{2}{ }^{*}$ kiirgusest Ne kristallis moodustab kuum luminestsents. 


\section{VIBRATIONAL RELAXATION OF QUASIMOLECULES IN THE CRYSTALS OF NOBLE GAS ELEMENTS}

A new quantum mechanical model of vibrational relaxation for molecular centers in the crystals is proposed. The most specific feature of the treatment is the presumption of weak coupling between the local center and environment atoms (dimensionless Stokes' losses $B \ll 1$, in contrast to the usually examined case $B \gg 1$ ). Another important precondition is $\Omega_{n} \gg \bar{\omega}$, i. e. the vibrational level spacing of the center under discussion greatly exceed the mean energy of phonons. It enables to introduce the so-called double adiabatic approximation. Within this approximation any vibrational level of the center is correlated with an adiabatic energy surface in the space of lattice normal coordinates. The tunnel transitions between the neighbouring adiabatic surfaces are induced by the corresponding operator of nonadiabaticity. A general formula for the transitions probabilities is derived: $\quad W_{n \rightarrow n-1}=\frac{A \cdot n}{\Omega^{3}\left(\Omega_{n}-2 \bar{\omega}\right)^{2}}$, where $A$ is a characteristic constant for given system and $n$ is the vibrational quantum number of the initial level of the center.

The preconditions of the theory are best of all fulfilled in the case of excimer centers $\mathrm{R}_{2}{ }^{*}$ in rare gas solids. Therefore, the theory is applied to the case of $\mathrm{Ne}_{2}{ }^{*}$ center in solid Ne. The vibrational transitions probabilities $W_{n \rightarrow n-1}$ are calculated and a reasonable quantitave explanation to the observed unusually intensive hot luminescence of this center is given. 